\title{
Psychological and behavioral intervention improves the quality of life and mental health of patients suffering from differentiated thyroid cancer treated with postoperative radioactive iodine- $|3|$
}

\author{
This article was published in the following Dove Press journal: \\ Neuropsychiatric Disease and Treatment \\ 2 May 2016 \\ Number of times this article has been viewed
}

\author{
Hong-Xia $\mathrm{Wu}^{1, *}$ \\ Hua Zhong ${ }^{2,3, *}$ \\ Yue-Dong $\mathrm{Xu}^{\prime}$ \\ Cui-Ping $\mathrm{Xu}^{4}$ \\ Ying Zhang ${ }^{5}$ \\ Wei Zhang' \\ 'Department of Endocrinology, \\ Shandong Provincial Qianfoshan \\ Hospital, Shandong University, \\ ${ }^{2}$ Department of Oncology, \\ Shandong University of Traditional \\ Chinese Medicine, ${ }^{3}$ Department \\ of Traditional Chinese Medicine, \\ Shandong Provincial Qianfoshan \\ Hospital, Shandong University, \\ ${ }^{4}$ Department of Nursing, Shandong \\ Provincial Qianfoshan Hospital, \\ Shandong University, Jinan, Shandong, \\ ${ }^{5}$ Department of Nursing, Tianjin Chest \\ Hospital, Tianjing, People's Republic \\ of China \\ *These authors contributed equally \\ to this work
}

\begin{abstract}
Background: We examined the effects of psychological and behavioral intervention on health-related quality of life and mental health among patients suffering from differentiated thyroid cancer (DTC) treated with postoperative radioactive iodine-131 (RAI).

Methods: Sixty patients with DTC, undergoing RAI, were randomly assigned to receive either conventional nursing $(n=30)$ or a 1-year psychological and behavioral intervention based on conventional nursing $(n=30)$. Health-related quality of life and mental health issues, depression, and anxiety were measured using the Quality of Life Core Questionnaire, Self-rating Depression Scale, and Self-rating Anxiety Score, respectively.

Results: After RAI treatment, patients in both groups showed improved functional capacities (ie, physical, role, cognitive, emotional, and social) and global quality of life, along with reduced depression and anxiety $(P<0.05)$. At 1 -year follow-up, compared with patients in the routine nursing group, those in the psychological and behavioral intervention group demonstrated greater improvements in functional capacities, global quality of life, and depression and anxiety symptoms $(P<0.05)$.

Conclusion: Psychological and behavioral interventions for patients with DTC undergoing RAI facilitated positive outcomes, suggesting that nursing care models that include psychological and behavioral interventions may be a complementary strategy for this patient population.

Keywords: psychological and behavioral intervention, radioactive iodine-131 treatment, differentiated thyroid cancer, health-related quality of life, depression, anxiety
\end{abstract}

\section{Introduction}

Thyroid cancer, the most common type of endocrine malignancy, is becoming increasingly prevalent worldwide and accounts for more deaths than all other endocrine cancers combined. ${ }^{1}$ In the United States, the yearly incidence rate has nearly tripled from 4.9 per 100,000 in 1975 to 14.3 per 100,000 in $2009 .{ }^{2}$ Similarly, from 1988 to 2009 , the incidence rate in the People's Republic of China has increased by 2.36 times with an average annual growth rate $5.92 \% .^{3}$ Differentiated thyroid cancer (DTC), arising from thyroid follicular cells that include both papillary and follicular histological types, accounts for $>90 \%$ of all thyroid cancers. ${ }^{4}$ Following the American Thyroid Association's guidelines for DTC management, standard care treatments include surgery, radioactive iodine-131 (RAI) treatment, and thyroid hormone suppression therapy. ${ }^{5}$ Among this standard regimen, the use of post-thyroidectomy adjuvant RAI is associated with dramatic decreases in locoregional recurrences, distant metastasis,

\footnotetext{
Correspondence: Hong-Xia Wu;

Wei Zhang

Department of Endocrinology, Shandong Provincial Qianfoshan Hospital, Shandong University, No 16766 Jingshi Road, Jinan, 250014 Shandong, People's Republic of China

Email junjie583।@sina.com; zhw08aoyun@163.com
} 
and disease-related mortality among patients with DTC. ${ }^{6}$ However, given the large radioactive iodine doses provided, patients receiving RAI need special protection (ie, being housed within radiation isolation wards); as a result, many patients fear radiation exposure, as well as loneliness and pessimism during isolation, which negatively affect their treatment adherence.

In modern medicine, health education has become an essential component of treatment. For instance, psychological nursing has been used to intervene in the lives of patients suffering from mental health issues, resulting in improvements to treatment adherence. Thus, we hypothesized that a specialist shared-care approach, including a psychological and behavioral intervention, would be a suitable alternative for improving treatment adherence among patients with DTC undergoing RAI. The present study used the Quality of Life Core Questionnaire (QLQ-C30), Self-rating Depression Scale (SDS), and Self-rating Anxiety Score (SAS) to compare the influence of psychological and behavioral intervention above and beyond a conventional nursing protocol on postoperative RAI for patients suffering from DTC.

\section{Patients and methods Study population}

This study conformed to the principles of the Declaration of Helsinki $\mathrm{i}^{7}$ and was approved by the Ethics Committee of Shandong Provincial Qianfoshan Hospital. Written informed consent was obtained, and participant confidentiality was strictly guarded. From 2013 to 2015, patients with DTC undergoing RAI treatment were recruited from the Department of Endocrinology, Shandong Provincial Qianfoshan Hospital. All patients with DTC were confirmed for their disease status through a pathological examination after a total thyroidectomy. Exclusion criteria were as follows: aged $<18$ years or $>70$ years, pre-existing psychiatric diagnosis, or undifferentiated thyroid cancer diagnosis. A single oral dose of RAI was administered to all enrolled patients.

\section{Intervention}

To compare the influence of psychological and behavioral intervention on patients with DTC undergoing RAI, patients were randomly divided into two groups: routine nursing group, and the psychological and behavioral intervention group. The routine nursing group underwent admission education, a medication guide, isolation protection education, and discharge guidance, as is conventionally performed for these patients. As for patients in the psychological and behavioral intervention group, in addition to the routine guidance, we provided a psychological and behavioral intervention protocol, as shown in Figure 1. Briefly, before RAI treatment, we conducted detailed interviews with each patient in a quiet ward, without any distraction, to better understand his or her inner feelings and nature of their psychological reaction to the treatment. We also administered a questionnaire survey to assess the degree of the patient's reactivity. Usually, uncertainties regarding the illness and prognosis may give rise to anxiety, depression, and other psychological problems among this patient population. Thus, we needed to establish appropriate communication channels with patients to develop a positive rapport and encourage cooperation. Meanwhile, we attempted to minimize any undesirable stimulation, remove harmful mental barriers, analyze the patients' thinking processes and mood changes, encourage them to express their true feelings, and enable them to safely express negative emotions (such as fear, anxiety, or depression) so as to guide them to bravely face the reality of their disease state. After the interviews, the collected data were processed and analyzed. After RAI, the patients were engaged in one-to-one conversations over the Internet, to address any further issues and provide emotional support while they were housed in the isolation ward. To further improve patients' social identity and sense of belonging, we regularly organized patient group meetings to allow them to express their own anticancer experiences. Thus, the patient's role transformed from an intervention recipient to an intervention provider, which was beneficial for improving his or her mental health status and life quality. During the entire intervention, we also cooperated with doctors to provide information regarding details of efficacious cases of DTC management, which helps promote methods for encouraging patients to undergo standardized RAI treatment regimen.

\section{QLQ-C30}

To evaluate health-related quality of life, the QLQ-C30 was used at baseline and 1 year after RAI. This internationally acknowledged questionnaire has been validated for assessing health-related quality of life among cancer patients participating in clinical trials, with an internal consistency of $>0.70 .{ }^{8}$ This questionnaire contains multi-item scales of functional capacities (ie, physical, role, cognitive, emotional, and social), symptom profiles (ie, fatigue, pain, and nausea/ vomiting), and global quality of life. For each assessment, the scores range from 0 to 100 , with a score of 100 representing perfect functional capacity and very strong symptom experiences. ${ }^{9}$ 


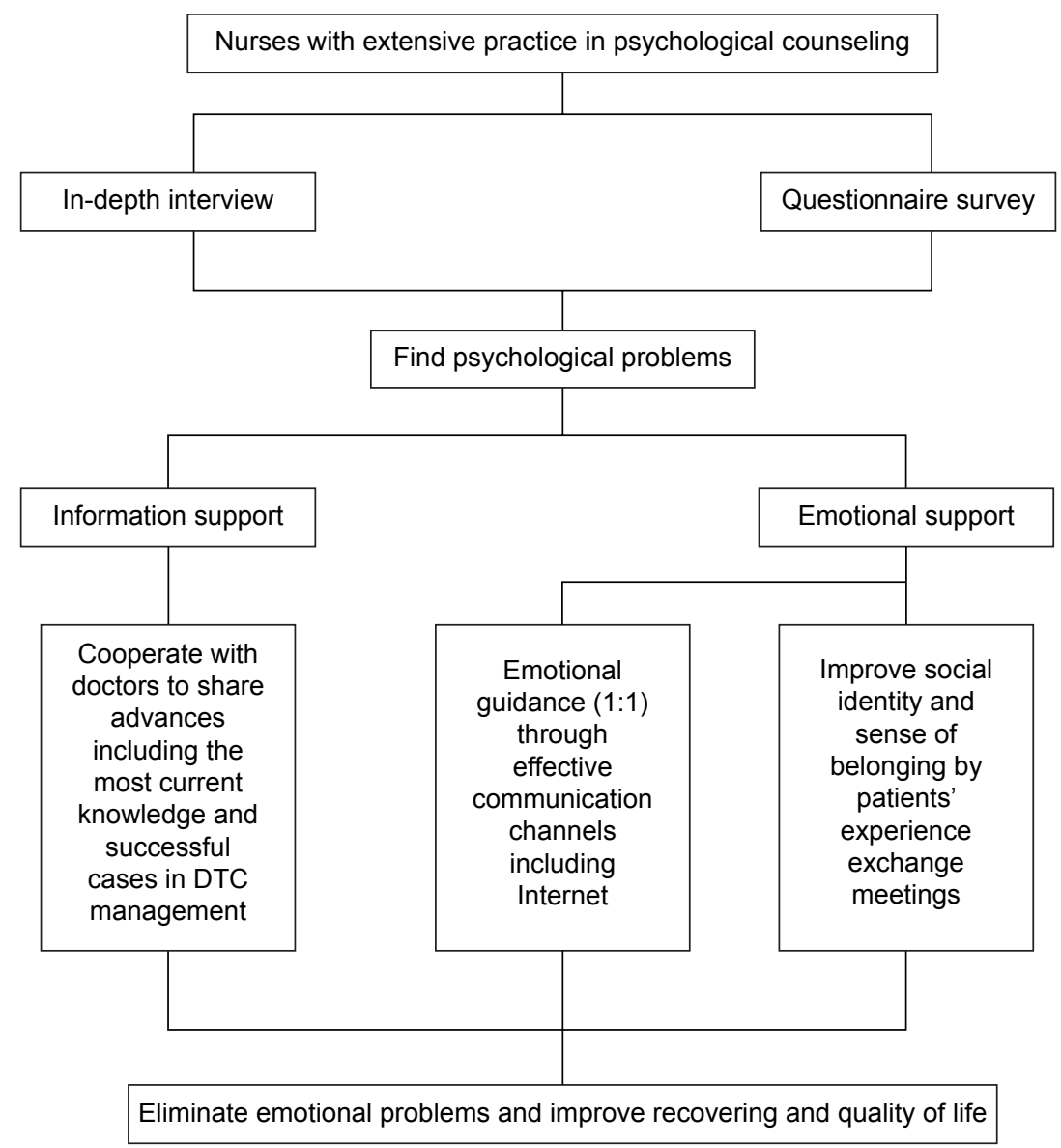

Figure I Psychological and behavioral intervention protocol.

Abbreviation: DTC, differentiated thyroid cancer.

\section{SDS and SAS}

To evaluate mental health status, the SDS and SAS were also utilized at baseline and 1 year after RAI. The SDS is a self-reported, 20-item measure of depressive symptoms, which has been confirmed as a valid and reliable instrument across several studies. ${ }^{10}$ The SAS is a self-reported measure of anxiety symptoms, which also contains 20 items. ${ }^{11}$ Item responses for both questionnaires are placed on a scale from 1 to 4 (1, rarely; 2 , some of the time; 3 , very often/often; 4 , almost always). The item scores were multiplied by 1.25 to obtain a standard 4-100 scale. The cutoff value for the SDS was 53 points, while for SAS it was 50 points, with higher scores reflecting more severe depressive or anxiety symptoms.

\section{Statistical analysis}

Statistical analysis was performed with Statistical Package for Social Science (version 12.0; SPSS Inc., Chicago, IL, USA). Quantitative variables are presented as mean \pm standard deviation. Student's $t$-test was used to analyze continuous, normally distributed variables. $P$-values $<0.05$ were considered statistically significant.

\section{Results}

As shown in Figure 2, 60 patients met our inclusion criteria and completed the 1-year follow-up. Of the 30 participants in the routine nursing group (male: 7, female: 23), 27 had papillary carcinoma and three had follicular thyroid carcinoma; the average age was $51.65 \pm 13.01$. For the remaining 30 participants in the psychological and behavioral intervention group (male: 8, female: 22), 26 had papillary carcinoma and four had follicular thyroid carcinoma; the average age was $50.92 \pm 10.58$. There were no significant differences in the sex, age, or pathological type between the two groups.

\section{Health-related quality of life}

As shown in Table 1, no QLQ-C30 subscales showed a significant difference between the two groups at baseline. After RAI, except for nausea/vomiting and pain, all other parameters (ie, physical functioning, role functioning, emotional functioning, 


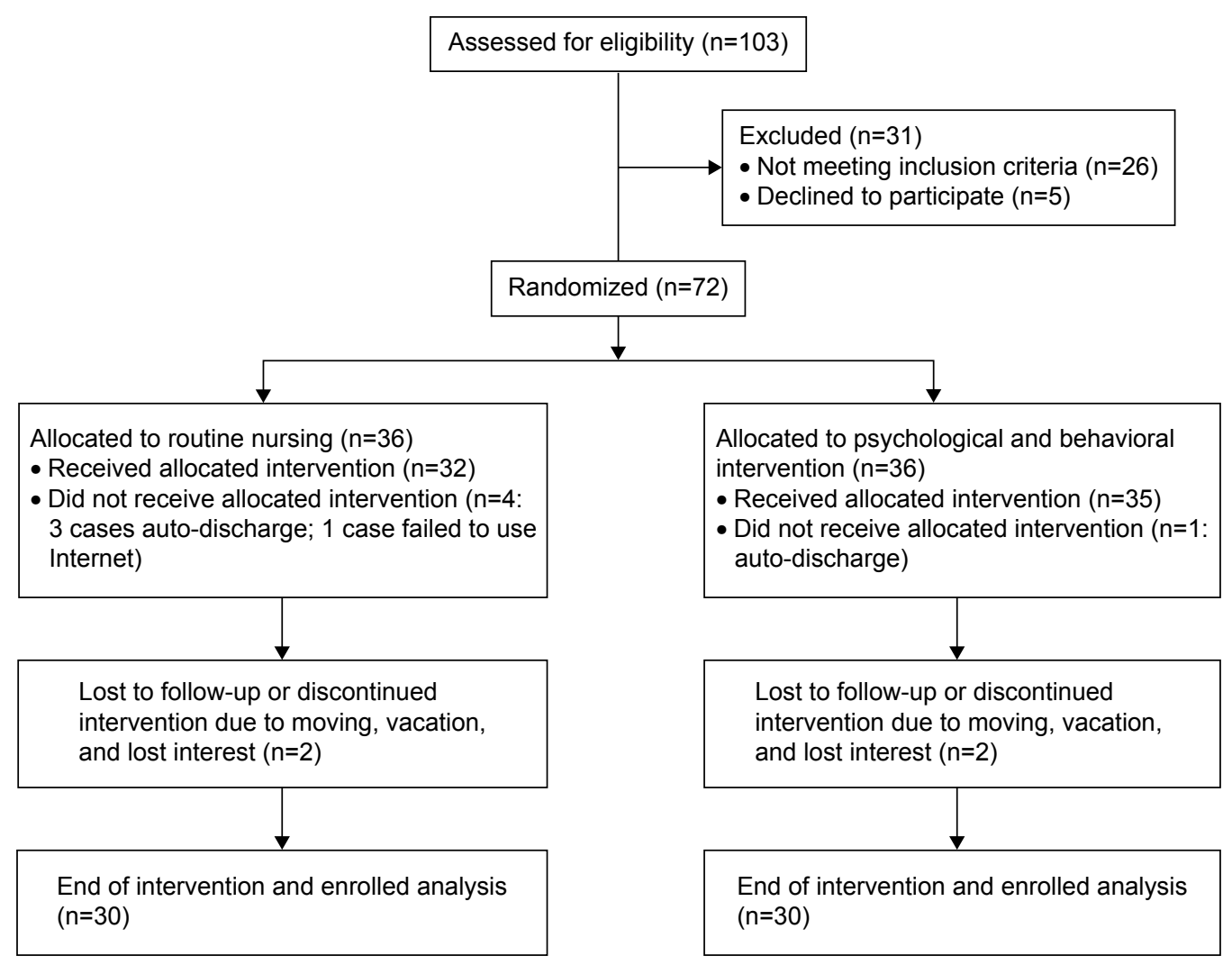

Figure 2 Flowchart showing enrolment criteria.

cognitive functioning, fatigue, and global quality of life) improved for both groups from before to after $\operatorname{RAI}(P<0.05)$. Interestingly, when comparing both groups after RAI, there was a significant increase on all functional scales (physical, role, cognitive, emotional, and social) and global quality of life in the intervention group $(P<0.05)$.

\section{Depression and anxiety}

As shown in Table 2, patients in both groups displayed higher SDS and SAS values at baseline compared to those at follow-up. Although there were no significant differences between the two groups before RAI, values were significantly higher than the standardized cutoffs (SDS: 53, SAS: 50, both $P<0.05$ ). Thus, all patients reported significant depression and anxiety symptoms prior to RAI treatment. At the 1-year follow-up, both groups reported significantly lower SDS and SAS scores (both $P<0.05$ ). Furthermore, this decline was more significant in the intervention group $(P<0.05)$. Therefore, above and beyond a conventional nursing care protocol, the inclusion of psychological and

Table I Comparison of two groups of QLQ-C30 before and I year after RAI treatment (mean \pm SD)

\begin{tabular}{|c|c|c|c|c|}
\hline \multirow[t]{2}{*}{ Subscales } & \multicolumn{2}{|c|}{ Routine nursing group } & \multicolumn{2}{|c|}{ Psychological intervention group } \\
\hline & Before RAI & I year after RAI & Before RAI & I year after RAI \\
\hline Physical function & $54.42 \pm 18.58$ & $65.28 \pm 19.89^{a}$ & $76.82 \pm 20.43$ & $76.82 \pm 20.43^{b, c}$ \\
\hline Role function & $52.90 \pm 20.85$ & $64.77 \pm 21.32^{\mathrm{a}}$ & $75.93 \pm 19.79$ & $75.93 \pm 19.79^{b, c}$ \\
\hline Emotional function & $40.12 \pm 17.91$ & $49.75 \pm 18.38^{a}$ & $65.43 \pm 19.21$ & $65.43 \pm\left. 19.2\right|^{b, c}$ \\
\hline Cognitive function & $55.72 \pm 23.47$ & $69.01 \pm 24.39^{\mathrm{a}}$ & $80.61 \pm 18.29$ & $80.61 \pm 18.29^{b, c}$ \\
\hline Social function & $39.24 \pm 19.67$ & $50.3 \mathrm{I} \pm 20.48^{\mathrm{a}}$ & $61.49 \pm 21.52$ & $61.49 \pm 21.52^{\mathrm{b}, \mathrm{c}}$ \\
\hline Fatigue & $40.45 \pm 17.57$ & $51.48 \pm\left. 19.3\right|^{a}$ & $61.74 \pm 18.56$ & $61.74 \pm 18.56^{\mathrm{b}, \mathrm{c}}$ \\
\hline Pain & $69.42 \pm 22.53$ & $69.24 \pm 23.31$ & $68.26 \pm 24.37$ & $68.26 \pm 24.37$ \\
\hline Nausea/vomiting & $74.03 \pm 25.16$ & $78.22 \pm 19.35$ & $80.97 \pm 18.39$ & $80.97 \pm 18.39$ \\
\hline Global quality of life & $50.98 \pm 24.56$ & $63.84 \pm 22.19^{a}$ & $75.39 \pm 20.75$ & $75.39 \pm 20.75^{\mathrm{b}, \mathrm{c}}$ \\
\hline
\end{tabular}

Notes: ${ }^{\text {a }}<<0.05$ vs values of before RAl in routine nursing group. ${ }^{b} P<0.05$ vs values of before RAl in psychological intervention group. ${ }^{c} P<0.05$ vs values of I year after RAI in routine nursing group.

Abbreviations: QLQ-C30, Quality of Life Core Questionnaire; RAI, radioactive iodine-13I; SD, standard deviation. 
Table 2 Comparison between two groups of SDS and SAS score before and I year after RAI treatment (mean \pm SD)

\begin{tabular}{llllll}
\hline Subscale & \multicolumn{2}{l}{ Routine nursing group } & & \multicolumn{2}{l}{ Psychological intervention group } \\
\cline { 2 - 3 } & Before RAI & I year after RAI & & Before RAI & I year after RAI \\
\hline SDS & $65.45 \pm 9.58$ & $59.87 \pm 8.69^{\mathrm{a}}$ & $67.76 \pm 7.43$ & $54.12 \pm 8.24^{\mathrm{b}, \mathrm{c}}$ \\
SAS & $69.48 \pm 8.42$ & $60.27 \pm 8.0 \mathrm{I}^{\mathrm{a}}$ & $68.65 \pm 8.36$ & $55.39 \pm 7.94^{\mathrm{b}, \mathrm{c}}$ \\
\hline
\end{tabular}

Notes: a $P<0.05$ vs values of before RAl in routine nursing group. ${ }^{b} P<0.05$ vs values of before RAl in psychological intervention group. ${ }^{c} P<0.05$ vs values of $\mathrm{I}$ year after $\mathrm{RAI}$ in routine nursing group.

Abbreviations: SDS, Self-rating Depression Scale; SAS, Self-rating Anxiety Score; RAI, radioactive iodine-I3I; SD, standard deviation.

behavioral intervention was more effective in alleviating distress reactions.

\section{Discussion}

We investigated the impact of a 1-year psychological and behavioral intervention, combined with routine nursing care, on the health-related quality of life and mental health status among patients with DTC receiving post-thyroidectomy adjuvant RAI treatment. As hypothesized, patients who were subjected to psychological and behavioral intervention reported significant improvement in their quality of life (as shown by their QLQ-C30 scores), as well as reduced depression and anxiety symptoms (as reflected in their SDS and SAS scores).

Patients with cancer often experience psychological problems due to heightened stress experience. ${ }^{12}$ Several studies have suggested that life quality can more accurately reflect the therapeutic effects of any intervention when compared to survival or fatality rates among patients with cancer. ${ }^{12}$ Therefore, psychological and behavioral intervention plays an important role in improving the life quality among patients with cancer. Studies have shown that if cancer patients can mobilize their bodily strength toward cancer resistance, this can help with recovery and remission. ${ }^{13}$ Thus, nurses, especially those with extensive practice and research experience in psychological counseling, can play a vital role in developing patients' positive attitudes toward treatment. In the present study, compared with patients receiving conventional nursing care, those provided with psychological and behavioral intervention reported higher QLQ-C30 scores and reduced SDS and SAS scores at 1-year follow-up. Thus, our results confirm that psychological and behavioral interventions, based on routine nursing care, can effectively improve the life quality and reduce mental health issues among patients with DTC undergoing RAI.

Depression and anxiety are common among patients with cancer. Since patients with DTC receiving RAI need special protection post radiation, they tend to display anxiety, loneliness, depression, and other negative mental states, especially during the isolation period. Nurses providing emotional support can help alleviate these psychological symptoms, which in turn may enhance the patients' abilities to respond to and actively cooperate with treatment. Therefore, it is necessary to create a systemic and consummate nursing care model for patients housed in isolation wards. In the present study, maintaining one-to-one communication with patients helped encourage them to express their negative emotions, as well as transfer any positive energy and enhanced support. Our results indicate that the psychological and behavioral intervention was effective in alleviating depressive and anxiety symptoms. Additionally, we regularly gathered the patients to organize meetings to express their experiences and exchange support with each other. During these meetings, by bringing out their own anticancer experiences, patients were able to transform themselves from the role of support receiver to one of support provider. This helped foster a more positive social identity and sense of belonging, which likely aided patients' improved mental health status and quality of life. Given knowledge regarding standard of living and long-term DTC survival, nurses must cooperate with doctors in order to educate patients to accept standardized treatment regimens, including RAI. It is possible that nursing care models with a psychological and behavioral intervention component can accomplish this goal.

A few study limitations should be noted. For instance, as this was a single-center study, with a relatively small number of patients, we may have issues with participant selection bias. Meanwhile, although this study was approved by the Ethics Committee of the Shandong Provincial Qianfoshan Hospital, we did not register it in any trial registry, which also means an important limitation. Furthermore, the investigation was completed 1 year after RAI. Before definitive conclusions can be drawn, these findings must be replicated with a larger sample and more research centers, and evaluations should be continued during longer term follow-ups.

\section{Conclusion}

We investigated aspects of health-related quality of life and mental health status among patients with DTC undergoing RAI. Specifically, we assessed whether a psychological and 
behavioral intervention strategy, in combination with a routine nursing care model, would help facilitate positive mental health and life quality outcomes. Given modern medical models (bio-social medicine) and improved living standards, improvements to patients' overall life quality and addressing patients' physiological and psychological requirements are necessary, above and beyond focusing on prolonged survival. ${ }^{14}$ Our results suggest that after 1 year of a consistent psychological and behavioral intervention, patients with DTC demonstrated improved life quality and mental health outcomes. Developing a reasonable and effective psychological nursing plan may be key to alleviating maladaptive symptom profiles, restoring a positive mental health status, and ultimately overcoming the disease. During daily nursing work, we are expected to fully consider a patient's worries and needs. Thus, it is important that a patient's mental health needs are included within a standard of nursing care, which should help better promote full patient recovery.

\section{Acknowledgments}

The authors would like to thank Editage (http://www. editage.cn/) for English language editing. This work was funded by the Natural Science Foundation (No ZR2009CQ023) and the Medical Science Development Plan (No 2009QZ025) of Shandong Province (both to WZ).

\section{Author contributions}

$\mathrm{H}-\mathrm{XW}, \mathrm{C}-\mathrm{PX}$, and WZ contributed to the study design. HZ, $\mathrm{Y}-\mathrm{DX}$, and $\mathrm{YZ}$ did the data collection and statistical analysis. $\mathrm{H}-\mathrm{XW}$ and WZ took the responsibility and are accountable for all aspects of the work in ensuring that questions related to the accuracy or integrity of any part of the work are appropriately investigated and resolved. All authors were involved in the critical revising of the manuscript and final approval of the proof published.

\section{Disclosure}

The authors report no conflicts of interest in this work.

\section{References}

1. Wartofsky L. Increasing world incidence of thyroid cancer: increased detection or higher radiation exposure? Hormones (Athens). 2010;9(2): 103-108.

2. Davies L, Welch HG. Current thyroid cancer trends in the United States. JAMA Otolaryngol Head Neck Surg. 2014;140(4):317-322.

3. Sun J, Xu X, Cai Q, Xu Y, Gu J. Epidemiological study on thyroid cancer in China. China Tumor. 2013;22:690-693.

4. Sherman SI. Thyroid carcinoma. Lancet. 2003;361(9356):501-511.

5. Haugen BR, Alexander EK, Bible KC, et al. 2015 American Thyroid Association Management guidelines for adult patients with thyroid nodules and differentiated thyroid cancer. Thyroid. 2016;26(1):1-133.

6. Patel SS, Goldfarb M. Well-differentiated thyroid carcinoma: the role of post-operative radioactive iodine administration. J Surg Oncol. 2013; 107(6):665-672.

7. World Medical Association declaration of Helsinki: recommendations guiding physicians in biomedical research involving human subjects. JAMA. 1997;277(11):925-926.

8. Hjermstad MJ, Fayers PM, Bjordal K, Kaasa S. Health-related quality of life in the general Norwegian population assessed by the European Organization for Research and Treatment of Cancer Core Quality-ofLife Questionnaire: the QLQ=C30 (+3). J Clin Oncol. 1998;16(3): 1188-1196

9. Aaronson NK, Ahmedzai S, Bergman B, et al. The European Organization for Research and Treatment of Cancer QLQ-C30: a quality-of-life instrument for use in international clinical trials in oncology. $J$ Natl Cancer Inst. 1993;85(5):365-376.

10. Zhang J, Xu CP, Wu HX, et al. Comparative study of the influence of diabetes distress and depression on treatment adherence in Chinese patients with type 2 diabetes: a cross-sectional survey in the People's Republic of China. Neuropsychiatr Dis Treat. 2013;9:1289-1294.

11. Ma YM, Ba CF, Wang YB. Analysis of factors affecting the life quality of the patients with late stomach cancer. J Clin Nurs. 2014;23(9-10): 1257-1262.

12. Robb SW, Benson K, Middleton L, Meyers C, Hébert JR. Mindfulnessbased stress reduction teachers, practice characteristics, cancer incidence, and health: a nationwide ecological description. $B M C$ Complement Altern Med. 2015;15:24

13. Shaw JM, Price MA, Clayton JM, et al. Developing a clinical pathway for the identification and management of anxiety and depression in adult cancer patients: an online Delphi consensus process. Support Care Cancer. 2016;24(1):33-41.

14. Grov EK, Dahl AA, Moum T, Fosså SD. Anxiety, depression, and quality of life in caregivers of patients with cancer in late palliative phase. Ann Oncol. 2005;16(7):1185-1191.
Neuropsychiatric Disease and Treatment

\section{Publish your work in this journal}

Neuropsychiatric Disease and Treatment is an international, peerreviewed journal of clinical therapeutics and pharmacology focusing on concise rapid reporting of clinical or pre-clinical studies on a range of neuropsychiatric and neurological disorders. This journal is indexed on PubMed Central, the 'PsycINFO' database and CAS,

\section{Dovepress}

and is the official journal of The International Neuropsychiatric Association (INA). The manuscript management system is completely online and includes a very quick and fair peer-review system, which is all easy to use. Visit http://www.dovepress.com/testimonials.php to read real quotes from published authors. 